\title{
Contact Mechanics of Rough Spheres: Crossover from Fractal to Hertzian Behavior
}

\author{
Roman Pohrt and Valentin L. Popov \\ Technische Universität Berlin, Straße des 17. Juni 135, 10623 Berlin, Germany \\ Correspondence should be addressed to Roman Pohrt; roman.pohrt@tu-berlin.de
}

Received 4 June 2013; Accepted 2 July 2013

Academic Editor: Dae-Eun Kim

Copyright ( 2013 R. Pohrt and V. L. Popov. This is an open access article distributed under the Creative Commons Attribution License, which permits unrestricted use, distribution, and reproduction in any medium, provided the original work is properly cited.

We investigate the normal contact stiffness in a contact of a rough sphere with an elastic half-space using 3D boundary element calculations. For small normal forces, it is found that the stiffness behaves according to the law of Pohrt/Popov for nominally flat self-affine surfaces, while for higher normal forces, there is a transition to Hertzian behavior. A new analytical model is derived describing the contact behavior at any force.

\section{Introduction}

Since Bowden and Tabor [1], it has been known that surface roughness plays a decisive role in contact, adhesion, friction, and wear. The main understanding of the contact mechanics of nominally flat rough surfaces was achieved in the middle of the 20th century due to works by Archard [2] and Greenwood and Williamson [3]. In the last years, contact mechanics of rough surfaces has once again become a hot topic [4-6]. Most of the previous work was devoted to investigation of nominally flat surfaces. For many tribological applications, however, the contact properties of rough bodies with macroscopically curved surfaces are of great interest. A first analysis of the contact problem including a curved but rough surface was given by Greenwood and Tripp [7]. They applied the Greenwood/Williamson (GW) model [3] of independent asperities with a Gaussian distribution to a parabolic shape. In this model, the roughness can be seen as an additional compressible layer. They calculated the mean pressures as a function of the radius and for low loads found a reduction in the maximum pressure and an enlargement of the apparent area of contact. For high loads, the indentation behavior found was Hertzian.

In the present paper, we will investigate the indentation of a rough sphere into an elastic half-space without the restrictions stemming from the GW model. We calculate the incremental normal stiffness of the contact, which not only determines the dynamic properties of a tribological system, but also its electrical and thermal conductivity $[8,9]$. In the interest of purity of results, we assume the bodies to be elastic at all scales and confine ourselves to self-affine roughness without cutoff. Contact stiffness of such surfaces has been recently studied in detail numerically and analytically $[10,11]$. We show that there is a pronounced crossover from the behavior which is typical for fractal surfaces $[12,13]$ to Hertzlike behavior [14], similar to GT [7]. Furthermore we derive an analytical approximation for the entire range of forces.

\section{Methods}

We consider a rigid rough spherical indenter with the radius $R$, which is approximated by a superposition of a parabolic shape $z_{p}(x, y)=\left(x^{2}+y^{2}\right) /(2 R)$ and a nominally flat random self-affine roughness $z_{r}(x, y)$ with the Hurst Exponent $H$ (Figure 1). The power spectrum $C_{2 \mathrm{D}}(q)$ of the randomly rough self-affine statistically isotropic surface has the form

$$
C_{2 \mathrm{D}}(q)=C_{0}(L q)^{-2 H-2},
$$

where $L$ is the system size and $q$ is the absolute value of the wave vector $\vec{q}$. The surface topography was calculated with 


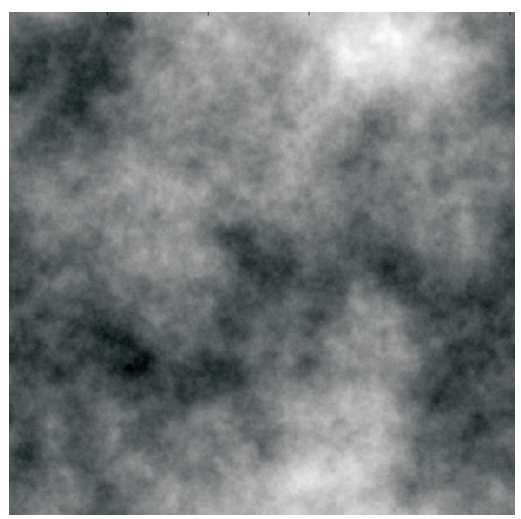

(a)

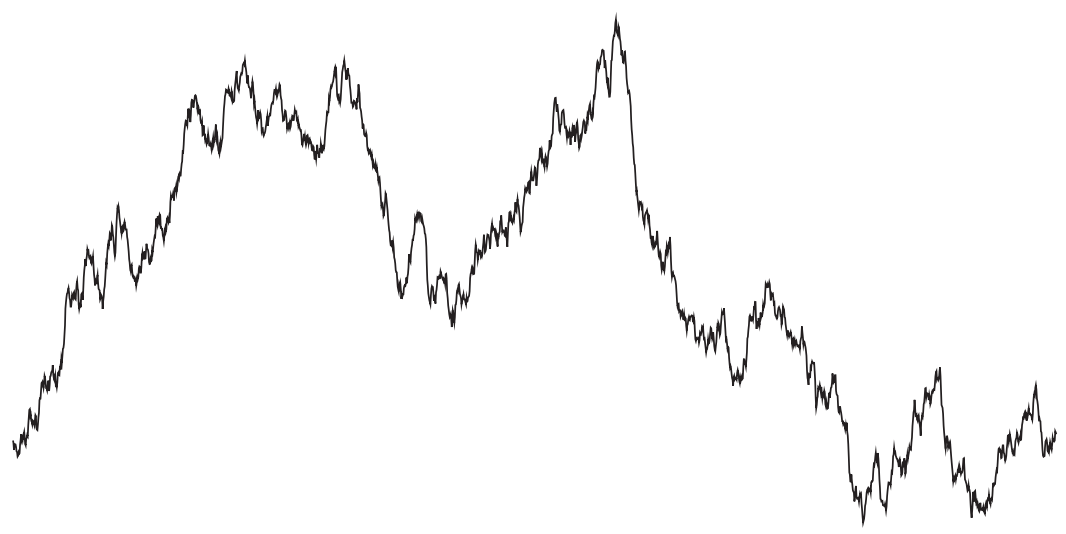

(b)

FIGURE 1: Graphical representations of a fractal rough surface with $H=0.6$ : (a) grayscale representation and (b) a cut through the surface.

the help of the power spectrum according to

$$
h(\vec{x})=\sum_{\vec{q}} B_{2 \mathrm{D}}(\vec{q}) \exp (i(\vec{q} \cdot \vec{x}+\phi(\vec{q})))
$$

with

$$
B_{2 \mathrm{D}}(\vec{q})=\frac{2 \pi}{L} \sqrt{C_{2 \mathrm{D}}(\vec{q})}=\bar{B}_{2 \mathrm{D}}(-\vec{q})
$$

and the phases $\phi(\vec{q})=-\phi(-\vec{q})$, which are randomly distributed on the interval $[0,2 \pi)$. All samples were generated on a grid with $513 \times 513$ discrete, evenly spaced points. A typical example of a self-affine surface with the Hurst exponent $H=0.6$ is shown in Figure 1, while Figure 2 shows a sample of the superposition of both sphere and roughness.

The indenter was pressed into an elastic half-space with the normal force $F$. The indentation depth and the configuration of the contact were calculated using the boundary element method with an iterative multilevel algorithm similar to $[15,16]$. The incremental normal stiffness $k$ was calculated by evaluating the differential quotient of force and indentation depth. All values were obtained by ensemble averaging of 50 surface realizations having the same power spectrum.

\section{Results and Discussion}

3.1. Numerical Results. Figure 3 shows the resulting dependency (red points). For small normal forces, the system is dominated by the roughness and the stiffness approaches the asymptotic dependence with the slope $(H+1)^{-1}$ characteristic for nominally flat fractal surfaces [12]. For higher normal forces, the influence of the roughness vanishes, and the system behaves like a smooth spherical Hertzian indenter (slope 1/3).

3.2. Analytical Estimation. In [12], it was shown that, for randomly rough self-affine surfaces, the contact stiffness is a power function of the normal force of the form

$$
k \propto F^{1 /(H+1)},
$$

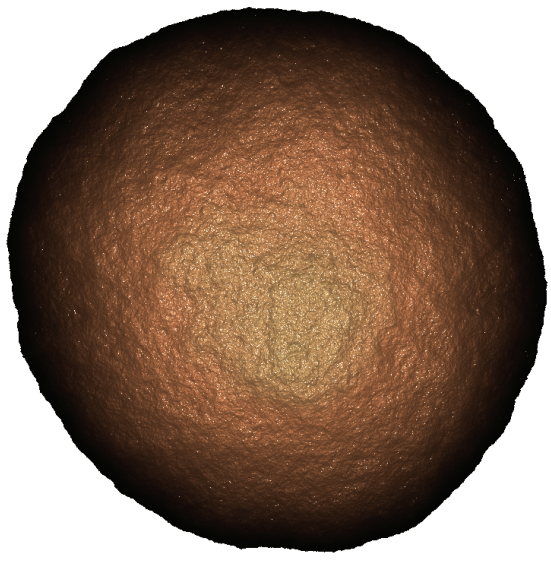

Figure 2: Numerically generated rough sphere, $H=0.7$.

with $0<H<1$. In [17], it was shown that this relation remains valid for Hurst exponents $0<H<2$ and that the only property needed for the validity of the scaling law (4) is the self-affinity of the surface which means that the surface appears undistinguishable from the original when viewed under an arbitrary magnification $\psi[15]$ :

$$
z_{r}^{\prime}(x, y)=\psi^{H} z_{r}\left(\frac{x}{\psi}, \frac{y}{\psi}\right) .
$$

Here it is not important whether the surface is randomly rough or just a simple axisymmetric profile with the same scaling properties [18]:

$$
z_{s}(r)=Q_{3 \mathrm{D}} \cdot|r|^{H} .
$$

Thus, with respect to the contact stiffness, the randomly rough, fractal, and self-affine surface can be replaced equivalently with a simple axisymmetric form (6). Of course, this equivalence only holds for the average values of multiple random rough realizations. In $[15,17]$, it was shown that, for the indentation of a rigid indenter having the shape (6) 


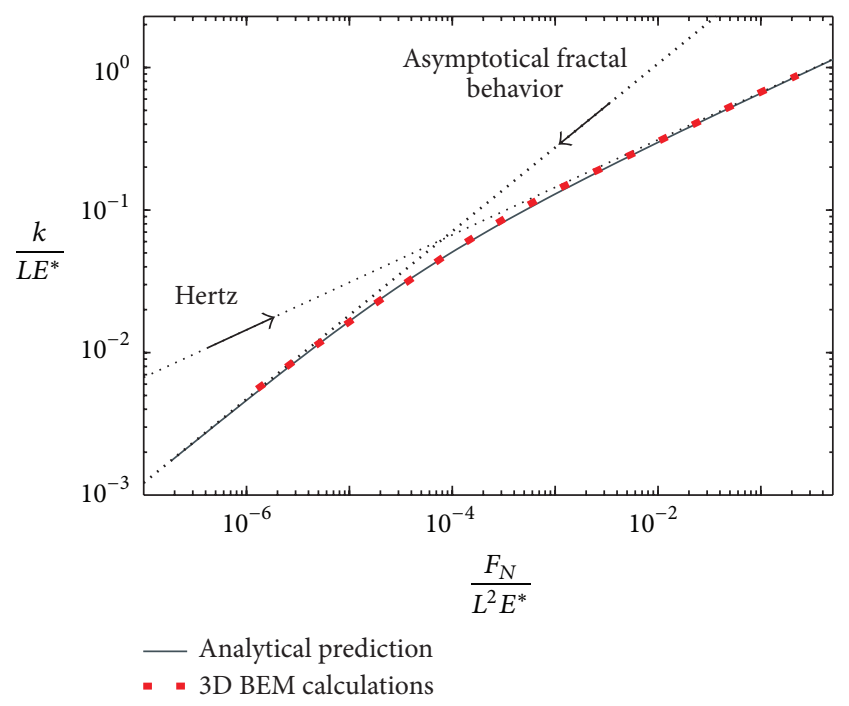

Figure 3: Normalized normal force $F_{N}$ versus normal stiffness $k$ for a spherical indenter, superimposed with a random roughness $\left(R=0.5, H=0.7, L=1, h_{\mathrm{rms}}=9 \cdot 10^{-3}\right)$. The red-dotted line is the result of a 3D boundary element study. The black line is the theoretical prediction according to (16). Furthermore, the asymptotical behavior of the plain roughness (11) and the smooth Hertzian case (9) are shown. The slope of the asymptotes is $1 / 3$ in the Hertzian case and $(H+1)^{-1}=0.5882$ for the roughness. The crossover occurs at $F_{\text {tr }} /\left(L^{2} E^{*}\right)=8 \times 10^{-5}$; see (17).

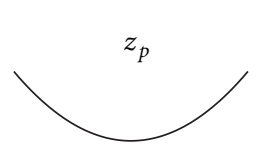

(a)

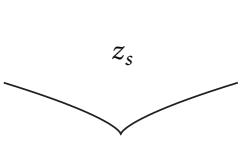

(b)

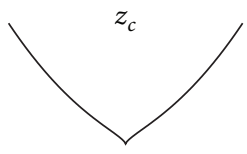

(c)
FIGURE 4: (a) Parabolic shape $z_{p}$; (b) indenter shape $z_{s}$ according to (6) with $H=0.7$; (c) superposition $z_{c}$ of both.

into an elastic half-space, the following relation of the normal contact stiffness $k$ and the normal force $F$ is valid:

$$
k=\frac{\partial F}{\partial d}=2 E^{*}\left(\frac{(H+1) F}{2 Q_{3 \mathrm{D}} E^{*} H \kappa(H)}\right)^{1 /(H+1)} \propto F^{1 /(H+1)},
$$

with

$$
\kappa(H)=H \int_{0}^{1} \frac{\xi^{H-1}}{\sqrt{1-\xi^{2}}} d \xi=\frac{\sqrt{\pi}}{2} \frac{H \Gamma(H / 2)}{\Gamma(H / 2+1 / 2)} .
$$

For example, the Hertzian contact is one particular case of self-affine surfaces with $H=2$ and $Q_{3 \mathrm{D}}=1 /(2 R)$, giving the classical result [14]:

$$
\frac{k}{E^{*}}=\left(6 R \frac{F}{E^{*}}\right)^{1 / 3}
$$

or, resolved with respect to the force,

$$
\frac{F}{E^{*}}=\frac{1}{6 R}\left(\frac{k}{E^{*}}\right)^{3}
$$

The same is true for fractal rough surfaces $[12,15]$ :

$$
\frac{k}{E^{*} L}=\zeta(H)\left(\frac{F}{E^{*} h L}\right)^{1 /(H+1)} \propto F^{1 /(H+1)}
$$

or

$$
\frac{F}{E^{*}}=h L\left(\frac{k}{\zeta(H) E^{*} L}\right)^{H+1} .
$$

The constant $\xi(H)$ must be (until now) found from numerical calculations. Latest studies suggest that

$$
\xi(H) \approx \frac{1.7}{H+1} .
$$

Comparison of (7) and (11) shows that the contact properties of a self-affine roughness and a corresponding axissymmetric profile can be made identical if the following prefactor $Q_{3 \mathrm{D}}$ in (6) is chosen:

$$
Q_{3 \mathrm{D}}=\left(\frac{2}{\zeta L}\right)^{H+1} \frac{(H+1) L h}{2 H \kappa(H)} .
$$

Using this analogy between randomly rough surfaces and axis-symmetric profiles, we suggest the rough sphere to be modeled as a superposition of the parabolic shape $z_{p}$ with the equivalent rotationally symmetric shape $z_{s}$ :

$$
z_{c}(r)=\frac{r^{2}}{2 R}+\left(\frac{2}{\zeta L}\right)^{H+1} \frac{(H+1) L h}{2 H \kappa(H)}|r|^{H} .
$$

Figure 4(c) shows a cut through the resulting threedimensional, rotationally symmetric indenter shapes.

As the contact stiffness depends only on the current contact radius [17], the superposition principle is valid for forces (10) and (12) at the given contact stiffness:

$$
F(k)=E^{*}\left[\frac{1}{6 R}\left(\frac{k}{E}\right)^{3}+\operatorname{Lh}\left(\frac{k}{\zeta E L}\right)^{H+1}\right] .
$$

For small values of the normal force or the contact stiffness we expect to see behavior according to (11), while at higher forces the Hertzian behavior (9) is predicted. The crossover is expected to take place at the intersection of both asymptotic curves:

$$
\frac{F_{\text {tr }}}{E^{*}}=\left[\zeta(H)^{3 H+3} h^{-3} L^{3 H}(6 R)^{-H-1}\right]^{1 /(H-2)} .
$$

The dependence (16) is shown in Figure 3 together with the numerical results of the Boundary Element Method. The solid line is the theoretical prediction according to (16). Furthermore, the asymptotical behavior of the plain roughness (11) and the smooth Hertzian case (9) are shown. The slope of the asymptotes is $1 / 3$ in the Hertzian case and $1 /(H+1)$ for the roughness.

\section{Conclusions}

We investigated the normal contact problem of a sphere with a self-affine roughness by means of the BEM and suggested 
a simple analytical method of calculating the contact stiffness for arbitrary normal forces. The method can be applied without changes to indentation of arbitrary bodies of revolution with self-affine roughness with any Hurst exponent in the range $0<H<2$.

In the present paper, we considered the contact partners to be elastic at all scales and confined ourselves to self-affine roughness without cutoff. For this class of contact problems, we have shown the validity of the superposition principle for profiles. The consideration of roughness covering all scales including the system size means no real restriction of the proposed approach, as a generalization to a more general case of surfaces having multifractal roughness or arbitrary power spectrum is straightforward.

In [17] it has been shown that the GW model is valid for surfaces with $H \leq 0$. We can thus compare the results from Greenwood and Trip to the current findings by assuming $H=0$. In this case, (16) predicts a linear dependence between normal force and contact stiffness just like GT although in the latter, this is not stated explicitly. The transition to Hertzian behavior occurs at

$$
\frac{F_{\mathrm{tr}}}{E^{*}}=\lambda h^{3 / 2} R^{1 / 2}
$$

with $\lambda \approx 1.1$ (17) or $\lambda \approx 1.41$ (GT, [7]), respectively.

Another insight can be gained from the comparison of both results. In the original GT paper [7], the apparent contact area was found to grow larger with added roughness compared to the smooth Hertzian case. Bearing in mind that the contact radius is proportional to the contact stiffness in concentrated contacts, one could expect the contact stiffness to grow larger as well. However the current results show that this is not the case. For loads smaller than the characteristic transition force (17), the added roughness will always lead to a decrease in the contact stiffness, compared to the smooth Hertzian case; see Figure 3.

The assumption of elasticity on all scales does not play a crucial role in the current findings. The reason is that the contact stress at any particular scale has the order of magnitude $\sigma \approx E^{*} \nabla z / 2$, where $\nabla z$ is the rms surface gradient corresponding to this scale [19]. The breakdown of the elastic behavior occurs therefore at the smallest scales corresponding to the largest rms surface gradient. The contact stiffness, on the contrary, is determined by the large wavelength in the power spectrum of the surface roughness and is not sensitive to the occurrence on the atomic scale.

\section{Acknowledgments}

The authors are grateful for a discussion with Lars Pastewka. This material is based upon work supported by the Deutsche Forschungsgemeinschaft (DFG, Grant no. PO 810/24-1).

\section{References}

[1] F. P. Bowden and D. Tabor, The Friction and Lubrication of Solids, Clarendon Press, Oxford, UK, 1986.
[2] J. F. Archard, "Elastic deformation and the laws of friction," Proceedings of the Royal Society A, vol. 243, no. 1233, pp. 190205, 1957.

[3] J. A. Greenwood and J. B. P. Williamson, "Contact of nominally flat surfaces," Proceedings of the Royal Society A, vol. 295, no. 1442, pp. 300-319, 1966.

[4] S. Akarapu, T. Sharp, and M. O. Robbins, "Stiffness of contacts between rough surfaces," Physical Review Letters, vol. 106, no. 20, Article ID 204301, 4 pages, 2011.

[5] R. Buzio, C. Boragno, F. Biscarini, F. Buatier De Mongeot, and U. Valbusa, "The contact mechanics of fractal surfaces," Nature Materials, vol. 2, no. 4, pp. 233-236, 2003.

[6] C. Campana, B. N. J. Persson, and M. H. Müser, “Transverse and normal interfacial stiffness of solids with randomly rough surfaces," Journal of Physics, vol. 23, no. 8, Article ID 085001, 2011.

[7] J. A. Greenwood and J. H. Tripp, "The elastic contact of rough spheres," ASME Journal of Applied Mechanics, vol. 34, no. 1, pp. 153-159, 1967.

[8] J. R. Barber, "Bounds on the electrical resistance between contacting elastic rough bodies," Proceedings of the Royal Society A, vol. 459, pp. 53-66, 2003.

[9] J. R. Barber, "Incremental stiffness and electrical contact conductance in the contact of rough finite bodies," Physical Review E, vol. 87, no. 1, Article ID 013203, 5 pages, 2013.

[10] R. Pohrt and V. L. Popov, "Normal contact stiffness of elastic solids with fractal rough surfaces," Physical Review Letters, vol. 108, no. 10, Article ID 104301, 4 pages, 2012.

[11] B. N. J. Person, "Contact mechanics for randomly rough surfaces," Surface Science Reports, vol. 61, no. 4, pp. 201-227, 2006.

[12] R. Pohrt, V. L. Popov, and A. E. Filippov, "Normal contact stiffness of elastic solids with fractal rough surfaces for one- and three-dimensional systems," Physical Review E, vol. 86, no. 2, Article ID 026710, 7 pages, 2012.

[13] R. Pohrt and V. L. Popov, "Investigation of the dry normal contact between fractal rough surfaces using the reduction method, comparison to 3D simulations," Physical Mesomechanics, vol. 15, no. 5-6, pp. 275-279, 2012.

[14] H. Hertz, "Ueber die berührung fester elastischer körper," Journal Für Die Reine Und Angewandte Mathematik, vol. 92, pp. 156-171, 1881.

[15] V. Popov, "Method of reduction of dimensionality in contact and friction mechanics: a linkage between micro and macro scales," Friction, vol. 1, no. 1, pp. 41-62, 2013.

[16] I. A. Polonsky and L. M. Keer, "A numerical method for solving rough contact problems based on the multi-level multisummation and conjugate gradient techniques," Wear, vol. 231, no. 2, pp. 206-219, 1999.

[17] V. L. Popov and M. Heß, Methode Der Dimensionsreduktion in Der Kontaktmechanik Und Reibung, Springer, 2013.

[18] N. Sneddon, "The relation between load and penetration in the axisymmetric boussinesq problem for a punch of arbitrary profile," International Journal of Engineering Science, vol. 3, no. 1, pp. 47-57, 1965.

[19] S. Hyun and M. O. Robbins, "Elastic contact between rough surfaces: effect of roughness at large and small wavelengths," Tribology International, vol. 40, no. 10-12, pp. 1413-1422, 2007. 

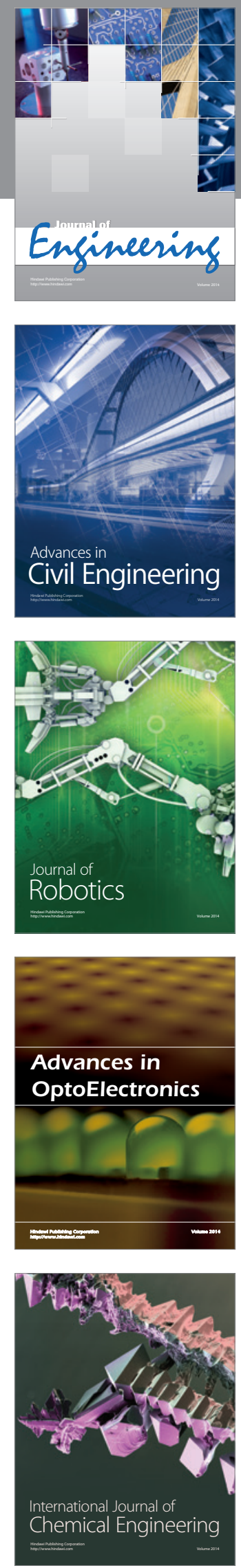

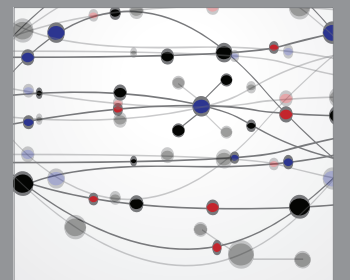

The Scientific World Journal
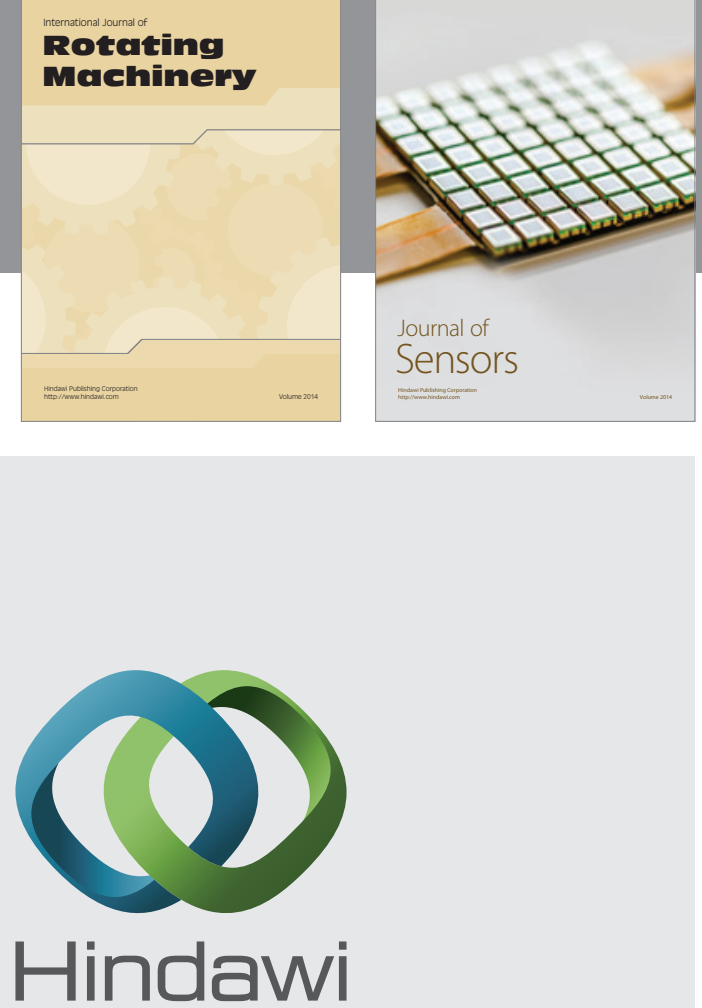

Submit your manuscripts at http://www.hindawi.com
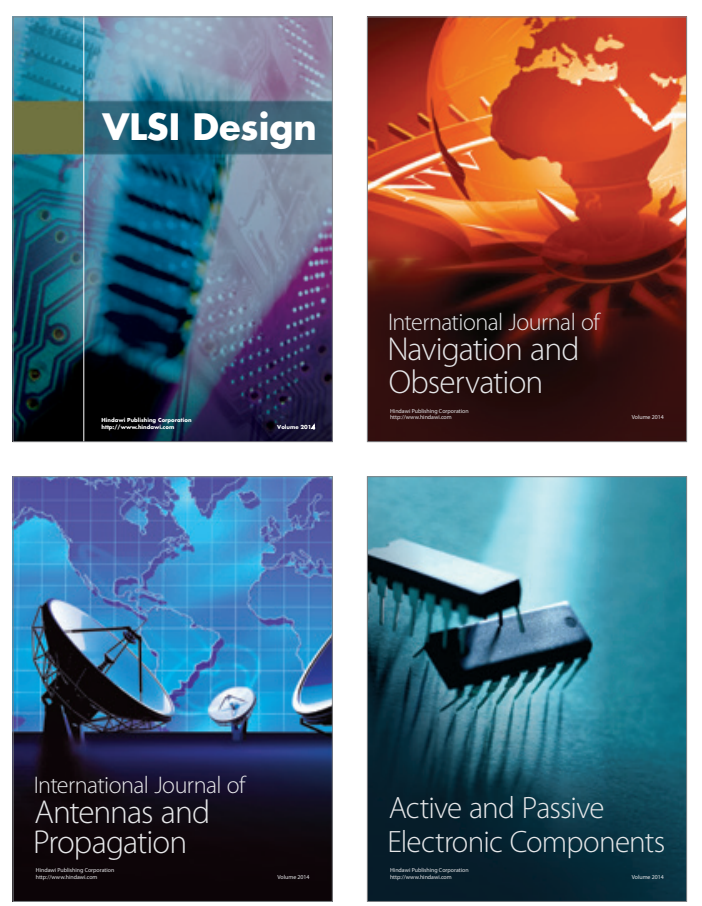
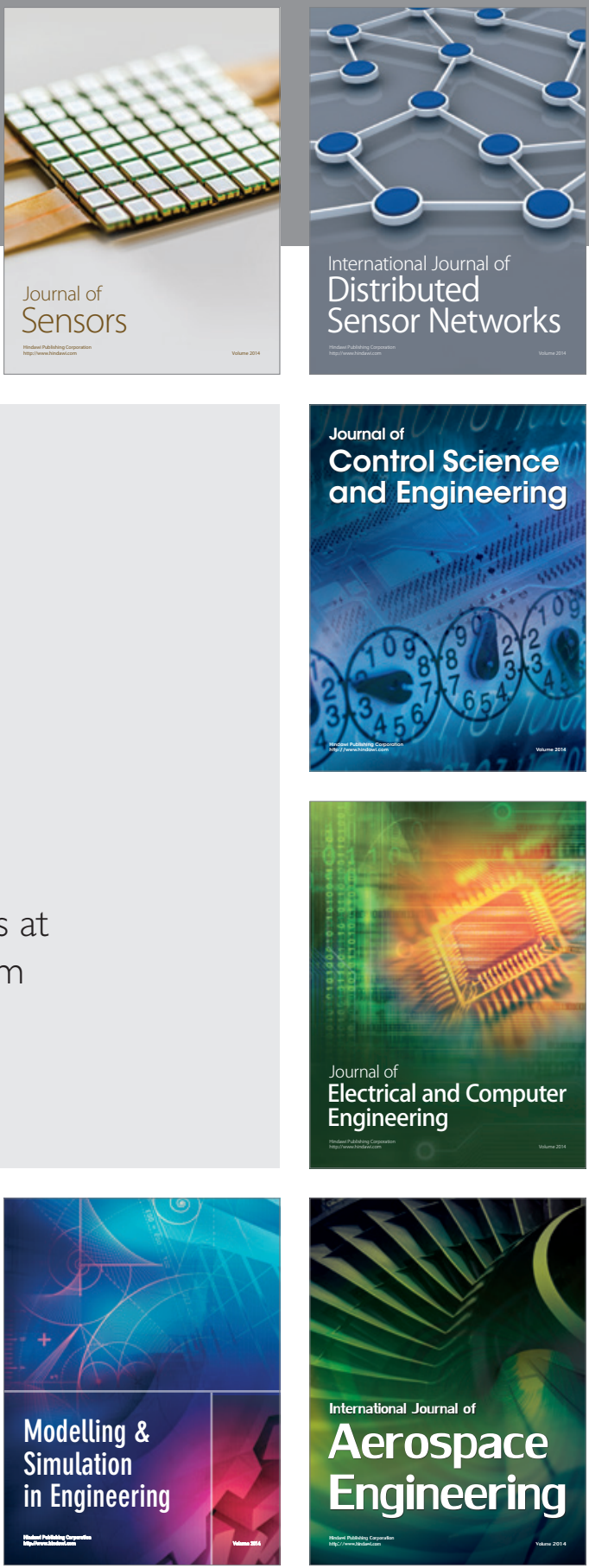

Journal of

Control Science

and Engineering
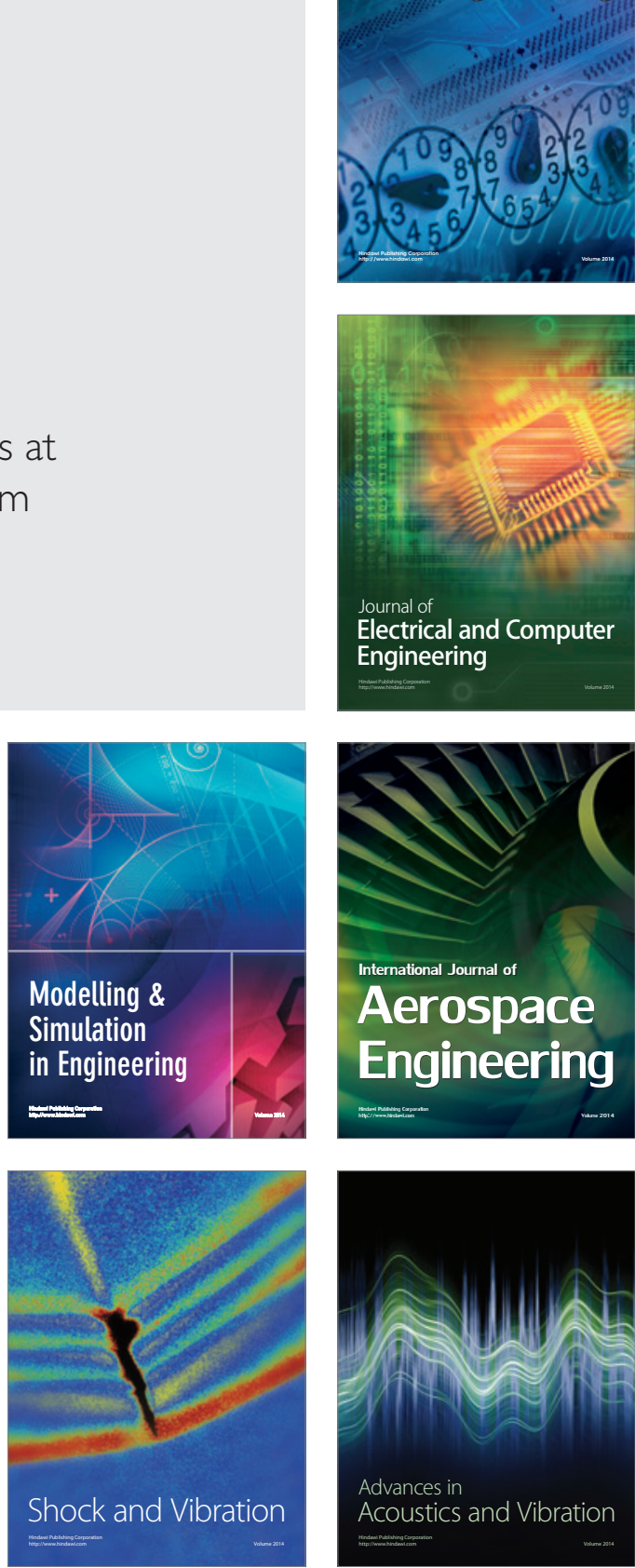